GA-A24069

\title{
NEXT-GENERATION PLASMA CONTROL IN THE DIII-D TOKAMAK
}

\author{
by \\ M.L. WALKER, J.R. FERRON, D.A. HUMPHREYS, R.D. JOHNSON, \\ J.A. LEUER, B.G. PENAFLOR, D.A. PIGLOWSKI, M. ARIOLA, A. PIRONTI, \\ and E. SCHUSTER
}




\section{DISCLAIMER}

This report was prepared as an account of work sponsored by an agency of the United States Government. Neither the United States Government nor any agency thereof, nor any of their employees, makes any warranty, express or implied, or assumes any legal liability or responsibility for the accuracy, completeness, or usefulness of any information, apparatus, product, or process disclosed, or represents that its use would not infringe privately owned rights. Reference herein to any specific commercial product, process, or service by trade name, trademark, manufacturer, or otherwise, does not necessarily constitute or imply its endorsement, recommendation, or favoring by the United States Government or any agency thereof. The views and opinions of authors expressed herein do not necessarily state or reflect those of the United States Government or any agency thereof. 
GA-A24069

\title{
NEXT-GENERATION PLASMA CONTROL IN THE DIII-D TOKAMAK
}

\author{
by
M.L. WALKER, J.R. FERRON, D.A. HUMPHREYS, R.D. JOHNSON, \\ J.A. LEUER, B.G. PENAFLOR, D.A. PIGLOWSKI, M. ARIOLA, ${ }^{\dagger}$ A. PIRONTI, ${ }^{\dagger}$ \\ and E. SCHUSTER ${ }^{\Delta}$
}

$\dagger_{\text {Associazione EURATOM/ENEA/CREATE }}$

$\triangle$ University of California, San Diego

This is a preprint of a paper presented at the 22nd Symposium on Fusion Technology, September 9-13, 2002, in Helsinki, Finland, and to be published in Fusion Engineering and Design.

\author{
Work supported by \\ the U.S. Department of Energy \\ under Contract DE-AC03-99ER54463 \\ and Grant DE-FG03-95ER54294
}

GA PROJECT 30033

OCTOBER 2002 


\section{ABSTRACT}

The advanced tokamak (AT) operating mode which is the principal focus of the DIII-D tokamak requires highly integrated and complex plasma control. Simultaneous high performance regulation of the plasma boundary and internal profiles requires multivariable control techniques to account for the highly coupled influences of equilibrium shape, profile, and stability control. This paper describes progress towards the DIII-D AT mission goal through both significantly improved real-time computational hardware and control algorithm capability. 


\section{INTRODUCTION}

DIII-D program needs and increased plasma control system (PCS) capabilities have synergistically driven demands for more varied shapes, more precise and comprehensive control, and more control of external systems such as neutral beams and ECH. The primary objectives for DIII-D control work are to provide improved capability for ongoing experimental operations, to prepare for future operational needs, and to make advances toward integrated control for Advanced Tokamak (AT) scenarios.

The ongoing upgrade of the PCS hardware [1] is motivated by the desire to improve execution of present controls and to prepare for planned and unplanned expansions of control capability. The present Intel i860 processors are becoming obsolete in terms of speed, memory, and maintainability. The replacement system has increased capability as well as improved long term extensibility and maintainability using primarily off-the-shelf components. In the near term, we will benefit from more accurate real-time equilibrium reconstructions and faster response for better shape control dynamics. Both the resistive wall mode (RWM) and advanced shape control efforts will benefit significantly from the shorter cycle times. A faster processor is essential when computation of the current profile is added to the real-time EFIT algorithm [2].

When properly tuned, the present PID algorithms provide good control. However, plasma shape oscillations and drift can still occur, especially when new shapes or shapes with modified current profiles are being controlled. Large changes of shape or profile within a single shot can move the plasma into regimes where the response is poorly matched to the control. Adjustments to control are presently made with 10 to 100 PID parameters, but the only guidance for these changes is via empiricism and intuition. As a result, new shapes require extensive machine time to tune controller gains and PID error signal conditioning. Changes to DIII-D geometry in the recent past have also pushed the plasma boundary further away from sensors and coils which makes shape control more challenging. Reduction in operator workload, better consistency in controlled response, and anticipation of future AT control capability all motivate the desire for more advanced shape control. 


\section{SHAPE CONTROL CHALLENGES ON DIII-D}

The initial step toward integrated control is implementation of a multivariable shape controller for routine operational use. Several problems make practical implementation of shape controllers on DIII-D and similar devices challenging: (1) Vertical stability and shape control share the same actuators, i.e. field shaping coils (F-coils) 2A, 2B, 6A, 6B, 7A, and 7B (Fig. 1). This is a particular problem for outer coils because these are the only coils which perform shape control for the outer plasma boundary. (2) Limitations on

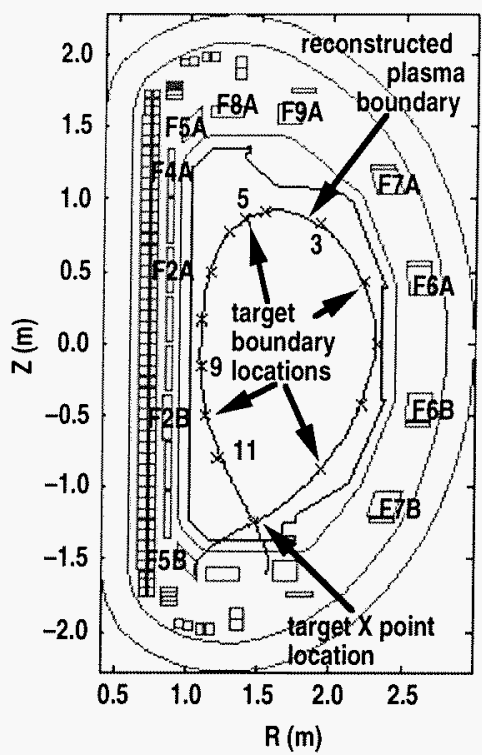
actuator voltage imply that commands to shaping power supplies (choppers) often

Fig. 1. DIII-D shape control target points and poloidal field coils ( $F$-coils).

saturate, particularly with large or fast disturbances. (3) Computational speed is insufficient to do both stabilization and shape control with the same controller. (4) Multiple current and voltage constraints imply that the range of accessible plasma equilibria is constrained. Attempts to reach equilibria outside these constraints can lead to exceeding coil current limits or saturation of actuators resulting in loss of control. (5) Changes in nonlinear plasma response during a discharge can degrade control performance by linear controllers. (6) The shape control power supply system is extremely nonlinear.

The present operational "decoupled" PID shape control [3] addresses each of these problems with varying degrees of success. Vertical stability and shape control loops operate on two separate time scales, about $60 \mu \mathrm{s}$ and $1 \mathrm{~ms}$, respectively. The vertical control coils are driven using a "frequency sharing" approach. Only derivative control is applied by an inner "stabilizing" loop which controls the high frequency behavior of the coils, while an outer shape control loop controls low frequency behavior. The inner loop does not actually stabilize, but reduces the growth rate sufficiently for the outer loop to stabilize the plasma. The present control with high gain, low order PID controllers provides a natural solution for actuator saturations. Actuators are allowed to saturate, then PID controllers quickly recover when supplies come out of saturation. During large disturbances, commands to vertical control coils are dominated by the vertical stability algorithm and boundary control near those coils degrades. Normally, when the plasma "settles down," authority of the low frequency behaviour of these coils is regained and 
boundary control returns to normal. However, for more unstable plasmas or for extended periods of programmed equilibrium change, vertical control commands will appear to alternate between minimum and maximum commands. In this case, the "average" command can appear unrelated to the shape command, so that there is essentially no active shape control from those actuators. 


\section{SUMMARY OF RECENT PROGRESS}

Recent efforts have focused on the upgrade of the PCS [1] and supporting controls needed for experimental operation [4]. The next-generation PCS hardware upgrade has been underway for two years. This upgrade extends the architecture of the PCS to much higher performance and flexibility, producing a factor of 10 or more increase in processor speed and adding a scalable network which accommodates a large number of real time cpus and peripherals. The first of two phases (Fig. 2) is complete. Three of the previous six VME based i860 real-time processors were replaced with PCI based Pentium 4 and Alpha computers. A 2 Gb/s Myrinet network was installed for interprocessor communication. This hybrid VME/PCI system was routinely used during the latter half of the 2002 experimental campaign. Calculations for real-time EFIT and shape control were performed on the new Alpha and Pentium processors, respectively.

Time-scale separation of vertical and shape control appears to be critical for DIII-D, since multivariable controllers can require significant computation. A method implemented for sharing actuators involves constructing a single linear controller which simultaneously stabilizes and provides control of vertical control coil currents with a fast cycle time. Figure 3 shows the closed loop system comprised of the DIII-D plant and stabilizing controller; the 6 coil currents F2A, F2B, F6A, F6B, F7A, and F7B are approximately controlled to a set of input reference values. This allows the system to act as an inner

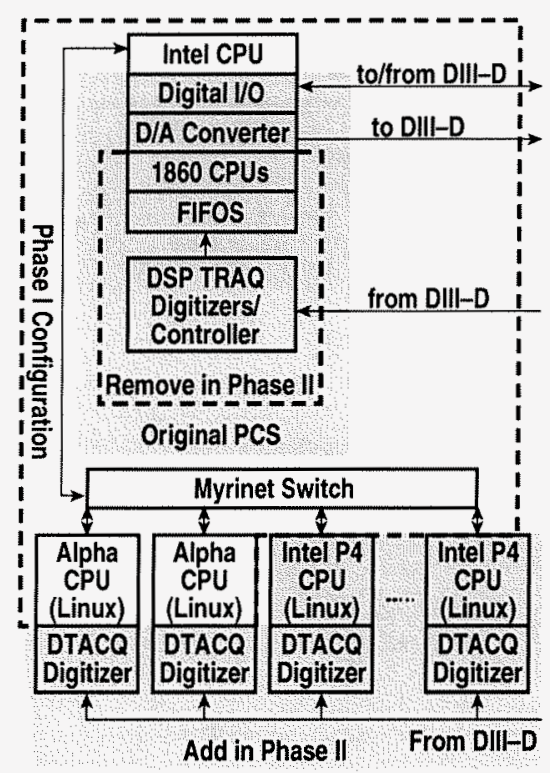

Fig. 2. Phased upgrade of the PCS. loop for shape control. It provides 6 coil current reference signals and up to 12 coil voltages as input actuators. By integrating control of the vertical control coils in a stabilizing controller, conflicts between shape and vertical control use of these coils are eliminated. "Frequency sharing" is accomplished explicitly with an $\mathrm{H}$-infinity loop-shaping design by weighting low frequencies to regulate coil currents and high frequencies to stabilize the plasma. The design technique ensures that the overall system remains robustly stable. A controller of this type was tested experimentally with excellent results. An outer loop shape control was subsequently implemented in the PCS and tested in simulation. The linear controller was developed using an H-infinity method. Nonlinear modifications of the control include logic to avoid current 
limits [3] and a variable loop gain to prevent controller windup. Some simulation results are shown in Fig. 4. The H-infinity method constructs a controller which provides a "weighted least squares" minimization of output errors. Since the $\mathrm{X}$-point (or strike point) control is typically of greatest concern to experimentalists at DIII-D, those errors were given greatest weight in the design. Thus, their tracking is superior to that of the gaps.

Because this is an "overdetermined" control problem (more control parameters

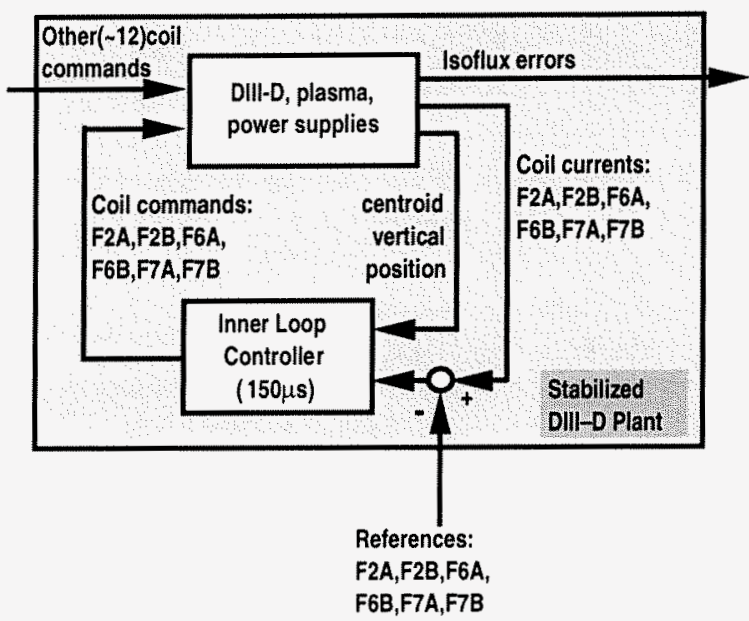

Fig. 3. Simultaneous stabilization and control of current in vertical control coils. than actuator degrees of freedom), individual target control points are not always accurately controlled. There are $18 \mathrm{~F}$-coils, but only 16 have power supplies. An additional sum of currents constraint reduces the linear degrees of freedom to 15 . This seems to match the set of 13 independent control points plus $\mathrm{X}$-point $\mathrm{R}$ and $\mathrm{Z}$ but this dimension count does not illustrate all constraints. Strong current and voltage limit constraints imply that the 15 control parameters cannot be simultaneously minimized. It is easy to choose a target shape incompatible with these constraints, even in steady-state. For example, a linear calculation for a particular equilibrium (shot $99339,1800 \mathrm{~ms}$ ) found that currents necessary to obtain the operator programmed target shape exceeded the DIII-D limits for 6 coils, some by more than a factor of 10 . Efforts by linear controllers to attain incompatible shapes can cause coils to exceed their current limits. Oscillations and difficulty with control in the simulated shot 900011 (Fig. 4) around $2.5 \mathrm{~s}$ are due to interaction of the linear control
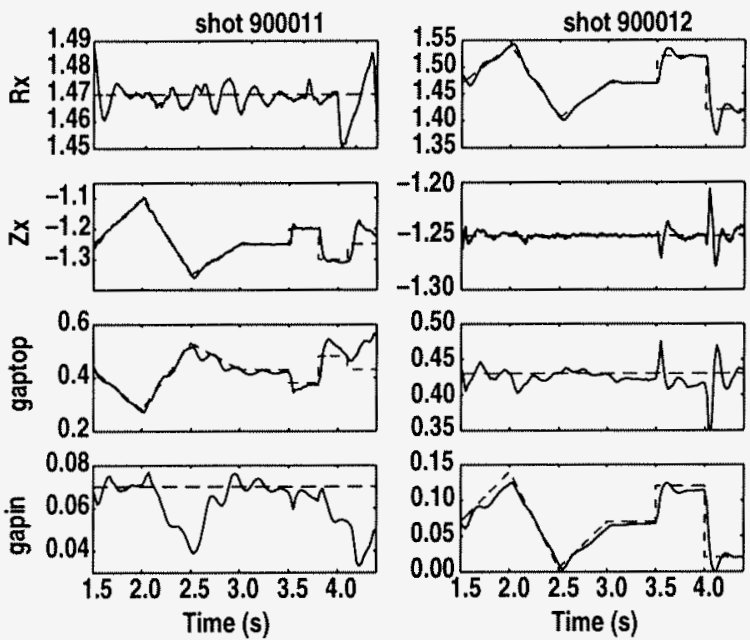

Fig. 4. Shape control simulation results (dashed $=$ requested, solid $=$ actual). All units in meters.

with nonlinear logic attempting to prevent coil F5B from approaching zero (a limit imposed by the shaping power supplies). The degradation of boundary control to prevent the shot from ending is the desired tradeoff here. Similarly, near the end of the shot, multiple coils have currents near limits and/or voltages in saturation. The simulated shot 900012 had fewer of these problems and consequently was more smoothly controlled. 
The choice was made at DIII-D to use an overdetermined system for reasons of flexibility. This stands in contrast to plans for future devices such as ITER where precise control of fewer parameters is required and little operator interaction is expected; shape evolution will be well studied in advance of device construction because of the severe consequences for loss of control. The present DIII-D multivariable controller implementation allows the operator to modify error weights for each control point and $\mathrm{X}$-point ( $\mathrm{R}$ and $\mathrm{Z}$ ) location. In simulation, increasing gain on errors for control points 9 and 10 (Fig. 1) by a factor of 10 reduced the inner gap error to less than half that shown for the 900011 results with no degradation in control of other parameters shown. The stability of the resulting modified controller relies on the inherent robustness of the shape controller. 


\section{FUTURE PLANS}

Phase II of the hardware upgrade is scheduled for completion in 2003. In this phase (Fig. 2), 32 channel simultaneous sampling PCI form DTACQ digitizers will take over data acquisition duties, several more Pentium processors will be installed, and most original PCS hardware will be converted for other purposes. The Digital I/O and Digital to Analog Convertor functions will remain VME based. Near-term plans to exploit the new computational capability include incorporation of MSE diagnostics into the real-time EFIT calculation to obtain accurate current profiles.

Careful tuning has been required to obtain good results from nonlinear methods used for dealing with actuator and plant limitations. Control analysis needed to define more systematic methods is ongoing. Work has also begun on algorithms to compute target equilibria more compatible with DIII-D device constraints. Real-time methods will still be required to handle cases where coil currents or voltages are at their limits, however, because of dependence of these equilibria on assumed current profiles and on models of the nonlinear DIII-D power system. 


\section{REFERENCES}

[1] B.G.Penaflor, et al., Fusion Eng.and Design 56-57, (2001), 739-742.

[2] J.R.Ferron,et al., Nucl.Fusion 38, (1988), 1055-1066.

[3] M.L.Walker, et al., Fusion Eng.and Design 56-57 (2001), 727-731.

[4] D.A. Humphreys, et al., "Advanced Tokamak Operation using the DIII-D Plasma Control System," this conference. 


\section{ACKNOWLEDGMENT}

Work supported by U.S. Department of Energy under Contract No. DE-AC03-99ER54463 and Grant No. DE-FG03-95ER54294. 